\title{
Combined therapeutic effects of bortezomib and anacardic acid on multiple myeloma cells via activation of the endoplasmic reticulum stress response
}

\author{
XIAOXIAN DONG ${ }^{1 *}$, YUNING LIAO $^{1 *}$, NINGNING LIU ${ }^{1,2}$, \\ XIANLIANG HUA ${ }^{1}$, JIANYU CAI ${ }^{1}$, JINBAO LIU ${ }^{1}$ and HONGBIAO HUANG ${ }^{1}$
}

${ }^{1}$ Protein Modification and Degradation Laboratory, Department of Pathophysiology, Guangzhou Medical University,
Guangzhou, Guangdong 510182; ${ }^{2}$ Guangzhou Research Institute of Cardiovascular Disease, The Second
Affiliated Hospital, Guangzhou Medical University, Guangzhou, Guangdong 510260, P.R. China

Received September 11, 2015; Accepted June 17, 2016

DOI: $10.3892 / \mathrm{mmr} .2016 .5533$

\begin{abstract}
Bortezomib (Bor), a proteasome inhibitor, has marked therapeutic effects in multiple myeloma (MM), and its synergistic effects with other anticancer agents have been widely investigated. In the present study, endoplasmic reticulum (ER) stress was the target of the treatment strategy; anacardic acid (AA) and Bor induce ER stress, resulting in apoptosis of multiple myeloma cells. AA/Bor combination therapy exhibited overt cytotoxicity in MM cells, by synergistically reducing cell growth and promoting cell death. Notably, expression levels of the stress-associated molecules binding protein, phosphorylated eukaryotic initiation factor $2 \alpha$, activating transcription factor 4 (ATF4) and CCAAT-enhancer binding protein homologous protein (CHOP) were increased following treatment. AA/Bor combination therapy-induced U266 cell cytotoxicity was partially reversed by ATF4 gene silencing and slightly enhanced by CHOP knockdown. The results of the present study suggest that AA/Bor combination may be a potential therapeutic strategy for MM treatment.
\end{abstract}

\section{Introduction}

Multiple myeloma (MM) is a clonal B-cell malignancy that primarily affects elderly individuals, accounting for $\sim 1 \%$ of all cancers $(1,2)$. Cancerous plasma cells accumulate in the

Correspondence to: Professor Jinbao Liu or Dr Hongbiao Huang, Protein Modification and Degradation Laboratory, Department of Pathophysiology, Guangzhou Medical University, 195 Dongfengxi Road, Guangzhou, Guangdong 510182, P.R. China

E-mail: jliu@gzhmu.edu.cn

E-mail: hhb800616@126.com

${ }^{*}$ Contributed equally

Key words: anacardic acid, bortezomib, endoplasmic reticulum stress, multiple myeloma bone marrow; the effects of this include hypercalcemia, renal failure, anemia and osteolytic bone lesions $(1,2)$. MM may be treated with the novel therapeutic agents, proteasome inhibitors and immunomodulatory drugs, which may be combined with conventional chemotherapeutics. However, almost all MM patients ultimately relapse, even when complete remission is achieved following initial therapy (2).

The majority of intracellular proteins are degraded by the ubiquitin-proteasome system (UPS) (3). Abnormal proteasome-dependent protein degradation is associated with the pathophysiology of multiple cancer types; therefore, it has been proposed that the selective inhibition of UPS may provide a novel strategy for the development of anticancer therapeutics (4-6). Notably, the proteasome inhibitor bortezomib (Bor) has been successfully developed for relapsed/refractory MM therapy. Bor has demonstrated a marked effect in MM patients; however, Bor resistance and its secondary side effects, including bone growth impairment, restrict the use of this therapy $(7,8)$. Adjuvant agents are therefore required to chemosensitize MM cells to Bor and achieve therapeutic efficacy with limited toxicity.

Bor treatment results in the aggregation of ubiquitinated proteins, endoplasmic reticulum (ER) stress and apoptotic cell death, via inhibition of $26 \mathrm{~S}$ proteasome activity. The proper folding of proteins prior to exit from the ER is ensured by quality control mechanisms; ER stress is triggered by improper protein folding and involves various signaling pathways collectively referred to as the unfolded protein response (UPR). Severe or prolonged ER stress promotes apoptotic cell death in the event that the UPR is unable to resolve the situation $(9,10)$. Various signaling pathways may modulate ER stress-induced programmed cell death. To date, three contributing UPR branches have been identified: Inositol-requiring enzyme 1, protein kinase RNA-like ER kinase (PERK) and activating transcription factor (ATF) 6 (11-13). PERK signaling induces eukaryotic initiation factor $2 \alpha$ (eIF $2 \alpha$ ) phosphorylation, enhancing ATF4 protein synthesis. The pre-apoptotic eIF2 $\alpha$-ATF4 signaling pathway involves binding protein (BiP), phosphorylated eIF2 $\alpha$, ATF4 and CCAAT-enhancer binding protein homologous protein (CHOP) activation (14-16). Heat 
shock protein 70 (HSP70; encoded by HSPA8), a member of the $70 \mathrm{kDa}$ HSPs family, is a primary chaperone involved in ER stress. HSP70 binds to and censors the folding status of substrate membrane proteins that are synthesized in the ER and transported to the cell surface via the conventional ER-to-Golgi secretion pathway (17).

Anacardic acid (AA; also referred to as 6-pentadecylsalicylic acid) is a constituent of the traditional medicinal plant Amphipterygium adstringens. Previous studies have revealed that AA exerts anticancer effects in various carcinomas $(18,19)$. Previous studies by our laboratory and others have demonstrated that AA induces ER stress $(20,21)$. In addition, it has been established that the ER stress inducer fenretinide sensitizes tumor cells to Bor-mediated killing (22). Therefore, the aim of the present study was to assess whether AA enhances the anticancer effects of Bor. AA was observed to significantly increase Bor activity via enhancing ATF4-dependent ER stress-associated caspase activation in vitro.

\section{Materials and methods}

Materials, reagents and antibodies. AA was manufactured by Sigma-Aldrich (St. Louis, MO, USA). Bor (Ben Venue Laboratories, Inc.; Boehringer Ingelheim Pharmaceuticals, Inc., Ridgefield, CT, USA) was used according to the manufacturer's instructions. Fetal bovine serum (FBS), RPMI-1640 and antibiotics were produced by Invitrogen; Thermo Fisher Scientific, Inc. (Waltham, MA, USA). Rabbit polyclonal anti-GAPDH antibody (clone, FL-335; catalog no. sc-25778; 1:500) was purchased from Santa Cruz Biotechnology, Inc. (Dallas, TX, USA). The following were purchased from Cell Signaling Technology, Inc. (Danvers, MA, USA): Rabbit monoclonal antibodies against nuclear poly (ADP-ribose) polymerase (PARP; clone, 46D11; catalog no. 9532; 1:1,000), eIF2 $\alpha$ (clone, D7D3; catalog no. 9079; 1:1,000), phospho-eIF2 $\alpha$ (Ser51; clone, D9G8; catalog no. 3398; 1:1,000), BiP (clone, C50B12; catalog no. 3177 ; 1:1,000), ATF4 (clone, D4B8; catalog no. 11,815; 1:1,000), caspase-3 (clone, 8G10; catalog no. 9665; 1:1,000) and caspase-8 (clone, D35G2; catalog no. 4790; 1:1,000); mouse monoclonal antibodies against caspase-9 (clone, C9; catalog no. 9508; 1:1,000) and CHOP (clone, L63F7; catalog no. 2895; 1:1,000); and a rat monoclonal antibody against HSP70 (clone 6B3; catalog no. 4873; 1:1,000). Rabbit polyclonal antibodies against active caspase-3 (catalog no. BS7004; 1:1,000), caspase-8 (catalog no. AP0358; 1:1,000) and caspase-9 (catalog no. BS7070; 1:1,000) were manufactured by Bioworld Technology, Inc. (St. Louis Park, NM, USA). Horseradish peroxidase (HRP)-conjugated goat anti-mouse $\operatorname{IgG}$ (catalog no. sc-395,763; 1:5,000), HRP-conjugated goat anti-rabbit IgG (catalog no. sc-2004; 1:5,000) and HRP-conjugated goat anti-rat IgG (catalog no. sc-2006; 1:5,000) were purchased from Santa Cruz Biotechnology, Inc. The enhanced chemiluminescence (ECL) kit was obtained from GE Healthcare Life Sciences (Chalfont, UK). Propidium iodide (PI) and Caspase-3 Activity and Annexin V-fluorescein isothiocyanate (FITC) Apoptosis Detection kits were manufactured by Nanjing Keygen Biotech Co., Ltd. (Nanjing, China).

Cell culture. U266 human myeloma cells were obtained from the American Type Culture Collection (Manassas, VA, USA) and cultured as previously described (21). AA and Bor were dissolved in dimethyl sulfoxide (DMSO) to a stock concentration of $50 \mathrm{mM}$, aliquoted and stored at $-80^{\circ} \mathrm{C}$. Prior to use, AA was diluted to 10,20 and $30 \mathrm{mM}$; Bor was diluted to 25,50 and $75 \mu \mathrm{M}$. During the treatment of each group, the corresponding drugs were diluted 1:1,000 in medium, added to the wells or plates and cultured at $37^{\circ} \mathrm{C}$ and $5 \% \mathrm{CO}_{2}$ for the indicated time.

3-(4,5-dimethylthiazol-2-yl-5-(3-carboxymethoxyphenyl)-2(4-sulfophenyl)-2H-tetrazolium (MTS) assay. Cytotoxicity was assessed by the MTS assay as described previously $(21,23)$. Exponentially growing cells were seeded into 96-well plates $(2,500 /$ well $)$ and incubated with drugs for $48 \mathrm{~h}$ prior to assessment with MTS.

Flow cytometric analysis of cell apoptosis. Exponentially growing cells were seeded into 6-well plates $\left(5 \times 10^{4} /\right.$ well) and incubated with drugs for $24 \mathrm{~h}$. Apoptosis was quantified in cells using Annexin V-FITC and PI double staining as previously described (24). Stained U266 cells were assessed by flow cytometry within $30 \mathrm{~min}$. The data was analyzed using FACSDiva software version 6.1.3 (BD Biosciences, Franklin Lakes, NJ, USA).

Caspase-3 activity evaluation. Exponentially growing cells were seeded into $6-\mathrm{cm}$ dishes $\left(1 \times 10^{6} /\right.$ well $)$ and incubated with drugs for $24 \mathrm{~h}$. Caspase-3 activity was determined in U266 cell lysates using a specific colorimetric assay kit according to the manufacturer's instructions. Following drug treatment, $1 \times 10^{6}$ cells were lysed with lysis buffer (Nanjing Keygen Biotech Co., Ltd.) and submitted to centrifugation $\left(10,000 \times \mathrm{g}, 4^{\circ} \mathrm{C}, 1 \mathrm{~min}\right)$. The supernatants were harvested and the enzyme-specific substrate was added at $37^{\circ} \mathrm{C}$ for $4 \mathrm{~h}$. The resulting product was quantified on a microplate reader at $405 \mathrm{~nm}$.

RNA interference. CHOP or ATF-4 genes were silenced using small interfering RNA (siRNA) technology as described previously (21). CHOP/GADD153 siRNA (catalog no. sc-35437), ATF4/CREB-2 siRNA (catalog no. sc-35112) and control siRNA (catalog no. sc-37007), purchased from Santa Cruz Biotechnology, Inc., were transfected separately into cells using Lipofectamine ${ }^{\circledR} 3000$ reagent (Invitrogen; Thermo Fisher Scientific, Inc.) according to the manufacturer's instructions.

Western blot analysis. Exponentially growing cells were seeded into 6-cm dishes (1 $1 \times 10^{6} /$ well) and incubated with drugs for $24 \mathrm{~h}$. Protein expression levels were determined as previously described $(25,26)$. Briefly, total protein extracts $(40 \mu \mathrm{g})$ from U266 cell lysates were resolved by $12 \%$ SDS-PAGE (100 V for $90 \mathrm{~min}$ ) and transferred onto polyvinylidene difluoride membranes. Membranes were blocked with 5\% milk, and following sequential incubations with primary and secondary antibodies, an ECL kit was used for protein detection. Blots were quantified with Image-Pro Plus software version 5.0 (Media Cybernetics, Inc., Rockville, MD, USA).

Combination index assessment. The effects of AA and Bor were assessed by evaluating the combination index (CI) using 
the Chou-Talalay method, as described previously $(26,27)$. A $\mathrm{CI}$ of $<1,1$ or $>1$ indicated synergistic, additive or antagonistic effects, respectively.

Statistical analysis. Data are presented as the mean \pm standard deviation. One-way analysis of variance was utilized to compare groups, with the least significant difference test being performed as a post hoc test. Statistical analyses were performed using SPSS software version 16.0 (SPSS, Inc., Chicago, IL, USA). $\mathrm{P}<0.05$ was considered to indicate a statistically significant difference.

\section{Results}

AA and Bor induce human myeloma U266 cell killing in a synergistic fashion. To assess if AA alone causes myeloma cell death, the effects of AA at various concentrations on MM cell viability were assessed. Cell viability was inhibited $<48 \%$ in U266 cells treated with $30 \mu \mathrm{M}$ AA as a monotherapy compared with DMSO treatment $(\mathrm{P}=0.001$; Fig. $1 \mathrm{~A})$. Based on these findings, 10, 20 and $30 \mu \mathrm{M}$ AA were co-administered for $48 \mathrm{~h}$ with 25, 50 and $75 \mathrm{nM}$ Bor. All CI results were $<0.8$, except one CI value of 0.809 (Fig. 1B), indicating synergy between these two agents in U266 cells. Doses of $20 \mu \mathrm{M}$ AA and $50 \mathrm{nM}$ Bor were chosen for subsequent experiments, as these doses were effective at reducing cell viability, but not to the extent that further analysis would be impossible.

AA sensitizes U266 cells to Bor-mediated caspase-dependent apoptosis. To investigate whether AA- and/or Bor-induced cytotoxicity correlated with cell death, myeloma cells were incubated with AA and/or BOR, and cell death was assessed using Annexin V/PI double staining. Co-administration of Bor and AA resulted in a significant increase in Annexin V and PI positive cells compared with monotherapy $(\mathrm{P}<0.001$; Fig. 2A and B), indicating that increased cell death was the result of Bor and AA combination therapy. The effects of combination therapy on cleavage of the apoptosis mediators, caspase and PARP, were investigated by western blotting. As presented in Fig. 2C, AA/Bor co-administration resulted in markedly enhanced cleavage of caspase-3, -8 and -9 , as well as PARP, compared with monotherapies. To confirm these results, caspase- 3 activity in cell lysates was assessed. AA/Bor combination therapy significantly increased caspase-3 activity compared with monotherapies $(\mathrm{P}<0.001$; Fig. 2D). These results suggested that AA sensitized U266 cells to Bor via caspase-dependent apoptotic cell death.

AA/Bor combination therapy amplifies ER stress. The effects of combination therapy on the UPR signaling pathway in U266 cells were analyzed by western blotting (Fig. 3). The expression levels of HSP70 $(\mathrm{P}=0.027)$ and $\mathrm{BiP}(\mathrm{P}=0.001)$ were significantly increased by $24 \mathrm{~h}$ compared with Bor monotherapy. Combination therapy induced increased protein expression levels of CHOP, phospho-eIF $2 \alpha$ and ATF4. These findings suggest that ER stress is involved in AA/Bor combination therapy-induced cell death.

Role of ER stress in AA/Bor combination therapy-mediated cytotoxicity. To identify UPR effectors involved in AA/Bor

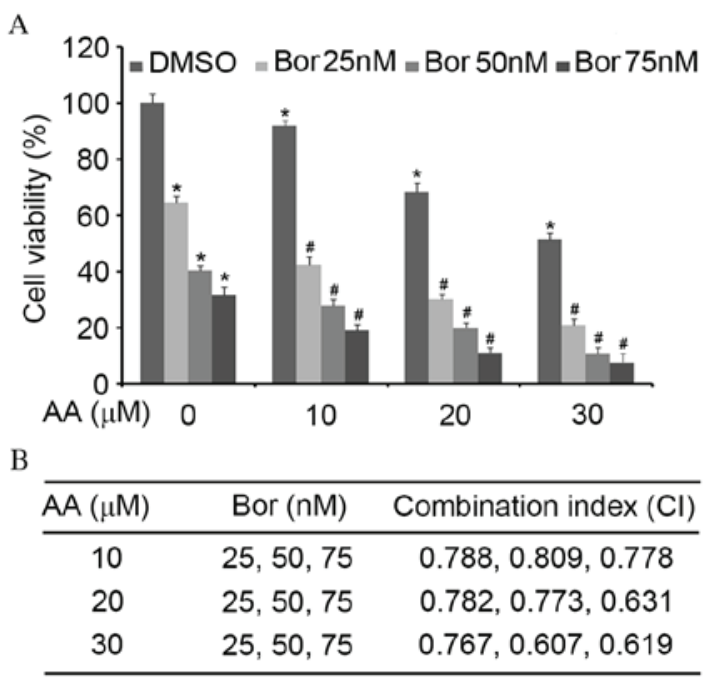

Figure 1. Effects of AA, Bor and combination therapy on cell viability. (A) U266 cells were incubated with Bor $(25,50$ or $75 \mathrm{nM})$ and AA $(10,20$, or $30 \mu \mathrm{M})$ as monotherapy or in combination for $48 \mathrm{~h}$. Cell viability was assessed using the 3-(4,5-dimethylthiazol-2-yl)-5-(3-carboxymethoxyphenyl)-2(4-sulfophenyl)-2H-tetrazolium assay. Cell viability was reduced by AA or Bor monotherapy, and by AA and Bor in combination, in a dose-dependent manner. Data are presented as the mean \pm standard deviation from three independent experiments. ${ }^{*} \mathrm{P}<0.05$ vs. DMSO; ${ }^{\#} \mathrm{P}<0.01$ vs. respective AA monotherapy. (B) Combination index values were determined, and revealed that AA and Bor act synergistically in U266 cells. AA, anacardic acid; Bor, bortezomib; DMSO, dimethyl sulfoxide.

combination therapy-mediated cell death, $\mathrm{CHOP}$ was silenced in U266 cells. Cells were then incubated for $24 \mathrm{~h}$ in the presence or absence of AA/Bor combination therapy. $\mathrm{CHOP}$ siRNA inhibited CHOP protein expression levels and slightly increased PARP cleavage in U266 cells incubated with combination therapy, compared to cells that received scrambled siRNA (Fig. 4A). In addition, CHOP silencing significantly increased the cytotoxicity of combination therapy compared with scrambled siRNA ( $\mathrm{P}=0.008$; Fig. 4B). These findings suggested that $\mathrm{CHOP}$ was not the primary UPR signaling pathway branch involved in U266 cell death mediated by AA/Bor combination therapy.

The role of ATF4 in AA/Bor combination therapy-mediated cell death was subsequently assessed. In contrast to CHOP repression, ATF4 silencing decreased PARP cleavage (Fig. 4C) and partially attenuated AA/Bor combination therapy-mediated cytotoxicity compared with scrambled siRNA $(\mathrm{P}=0.002$; Fig. 4D). These data indicate that ATF4-dependent ER stress contributed, at least partially, to AA/Bor combination therapy-mediated cytotoxicity.

\section{Discussion}

Various novel natural compounds have been reported to have synergistic anti-cancer cytotoxic effects when administered in combination with Bor $(26,28,29)$. Our previous study demonstrated that AA is a potent inducer of ER stress (21). Based on previous findings that the ER stress inducer fenretinide sensitizes tumor cells to killing by Bor (22), the effect of AA/Bor combination therapy on U266 cells in vitro was investigated, to examine the potential clinical application of AA. 


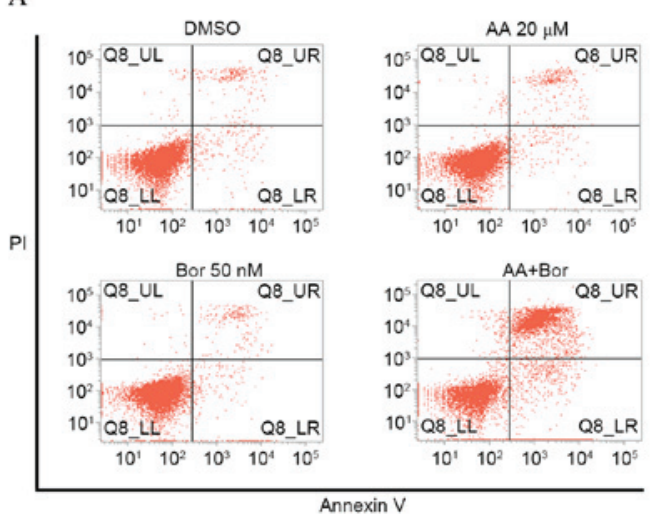

$\mathrm{C}$

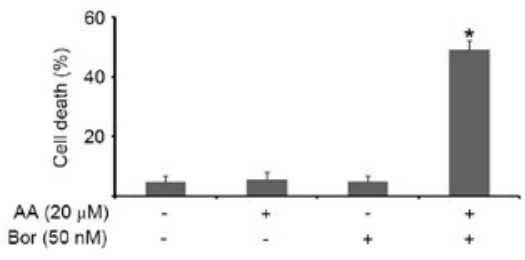

$\mathrm{D}$

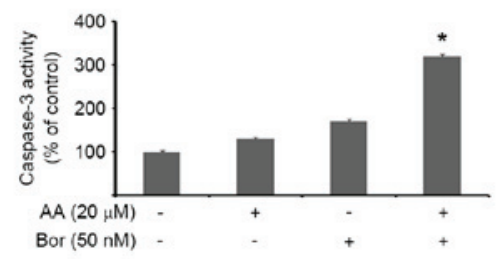

Figure 2. AA sensitizes U266 cells to Bor-induced cytotoxicity. U266 cells were incubated with AA $(20 \mu \mathrm{M})$, Bor $(50 \mathrm{nM})$ or combination therapy for $24 \mathrm{~h}$. (A) Cells were stained with Annexin V and propidium iodide. Representative flow cytograms are presented. Apoptotic cells were defined as those in the upper left, upper right and lower right quadrants. Co-administration of Bor and AA resulted in a significant increase in Annexin V and PI positive cells compared with monotherapy. (B) Flow cytometric analysis of (A), presented as the mean $\pm \mathrm{SD}(\mathrm{n}=3)$. " $\mathrm{P}<0.01$ vs. monotherapy. (C) Western blotting was performed to assess the expression levels of various proteins, with GAPDH serving as a loading control. Cleavage of caspase- $3,-8$ and -9 , and PARP, was increased following AA/Bor co-administration. (D) Caspase-3 activity was assessed in U266 cells by colorimetric assay, and was significantly increased upon AA/Bor combination therapy. Data are presented as the mean $\pm \mathrm{SD}(\mathrm{n}=3)$. ${ }^{*} \mathrm{P}<0.01$ vs. monotherapy. AA, anacardic acid; Bor, bortezomib; PI, propidium iodide; $\mathrm{SD}$, standard deviation; cas, caspase; PARP, poly (ADP-ribose) polymerase.

A

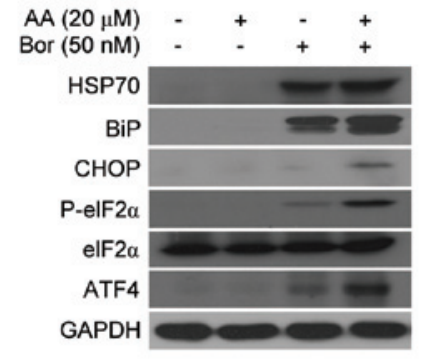

B
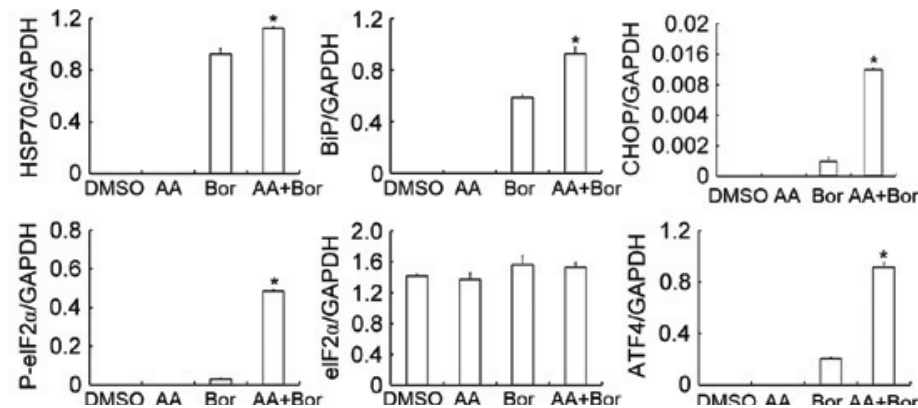

Figure 3. AA enhances Bor-induced ER stress. U266 cells were incubated with AA ( $20 \mu \mathrm{M})$, Bor $(50 \mathrm{nM})$ or combination therapy for $24 \mathrm{~h}$. (A) Western blotting was performed to analyze protein expression levels of HSP70, BiP, CHOP, P-eIF2 $\alpha$, eIF2 $\alpha$, ATF4 and GAPDH. (B) Protein bands were quantified and normalized to GAPDH. The protein expression levels of HSP70, BiP, CHOP, P-eIF2 $\alpha$ and ATF4 were significantly increased by AA/Bor combination therapy. ${ }^{*} \mathrm{P}<0.05$ vs. monotherapy. Data are presented as the mean \pm standard deviation $(\mathrm{n}=3)$. AA, anacardic acid; Bor, bortezomib; ER, endoplasmic reticulum; HSP70, heat shock protein 70; BiP, binding protein; CHOP, CCAAT-enhancer binding protein homologous protein; eIF $2 \alpha$, eukaryotic initiation factor $2 \alpha$; P, phosphorylated; ATF4, activating transcription factor 4; DMSO, dimethyl sulfoxide.

Inhibition of cell growth and promotion of apoptosis constitute the primary mechanisms underlying the cytotoxicity of cancer chemotherapeutics; therefore, the present study assessed these effects. AA or Bor alone inhibited cell viability in a dose-dependent manner. Notably, the combined inhibitory effects of AA and Bor on cell viability were markedly greater compared with those observed following $\mathrm{AA}$ and Bor monotherapies in vitro, with CI values $<0.8$. In addition, Bor and AA combination therapy significantly increased cancer cell apoptosis compared with AA or Bor treatment alone. Proteasome inhibition by Bor induces caspase activation; this constitutes an important mechanism 
A

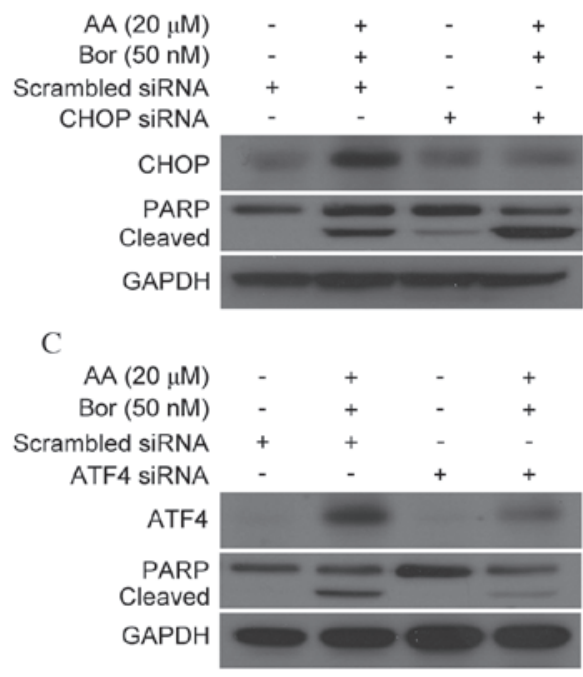

B
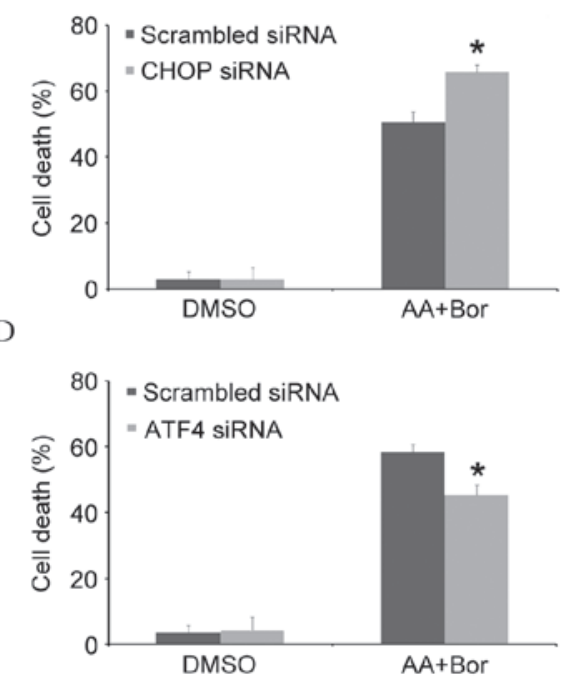

Figure 4. AA enhances Bor-mediated cytotoxicity involving ATF4 but not CHOP. CHOP-silenced U266 cells were incubated with AA (20 $\mu$ M), Bor (50 nM) or combination therapy for $24 \mathrm{~h}$. (A) Western blotting was performed to assess the protein expression levels of CHOP and PARP, with GAPDH serving as a loading control. (B) Apoptotic cell death was assessed by flow cytometry; CHOP silencing increased the cytotoxicity of combination therapy. ATF4-silenced U266 cells were incubated with AA $(20 \mu \mathrm{M})$, Bor $(50 \mathrm{nM})$ or combination therapy for $24 \mathrm{~h}$. (C) Western blotting was performed to assess the protein levels of ATF4 and PARP, with GAPDH serving as a loading control. (D) Apoptotic cell death was assessed by flow cytometry; ATF4 silencing decreased the cytotoxicity of combination therapy. Data are presented as the mean \pm standard deviation $(\mathrm{n}=3)$. ${ }^{*} \mathrm{P}<0.05$ vs. scrambled siRNA. AA, anacardic acid; Bor, bortezomib; CHOP, CCAAT-enhancer binding protein homologous protein; ATF4, activating transcription factor 4; PARP, poly (ADP-ribose) polymerase; siRNA, small interfering RNA; DMSO, dimethyl sulfoxide.

underlying Bor-induced cell death (30-32). In the present study, combined treatment with AA and Bor activated caspase-3, -8 and -9 , and induced PARP cleavage in U266 cells. AA/Bor co-administration promoted U266 apoptotic cell death via intrinsic (mitochondria-mediated; associated with caspase-9) and extrinsic (death receptor-mediated; associated with caspase-8) pathways, reflected by increased activation of caspase- $3,-8$ and -9 , alongside PARP cleavage.

Certain studies have demonstrated that Bor activates HSPs, including HSP90, HSP70 and HSP25, which are associated with Bor resistance $(33,34)$. Qi et al $(35)$ reported that inhibition of inducible HSP70 increases Bor-induced human bladder cancer cell cytotoxicity. In the present study, AA/Bor combination therapy in U266 cells was associated with increased HSP70 induction. These results support the notion that enhancing Bor-mediated HSP70 induction represents an attractive means of enhancing its activity.

Protein synthesis, folding and trafficking occurs primarily in the ER; thus, intensive ER stress results in cell death $(9,10)$. AA and Bor are ER stress inducers $(21,22)$; therefore, it was investigated whether combination therapy induced UPR signaling. BiP, CHOP, phospho-eIF2 $\alpha$ and ATF4 were all induced in U266 cells treated with AA and Bor. A previous study revealed that the ER stress-induced transcription factor ATF4 is a key mediator of Bor-induced cytotoxicity in neuro-ectodermal tumor cells, while CHOP is dispensable (14). Beck et al (36) reported that vemurafenib-induced melanoma cell death is associated with ATF4- but not CHOP-dependent ER stress, in agreement with our previous report (21). The effects of CHOP and ATF4 in promoting apoptosis were investigated in the present study. Consistent with previous reports, $\mathrm{CHOP}$ silencing failed to reduce the cytotoxic activity of combination therapy, and instead moderately enhanced this effect. However, ATF4 knockdown significantly reduced the cytotoxic effects of AA/Bor combination therapy. These findings demonstrate that ATF4 and CHOP are pro- and anti-apoptotic, respectively, in AA/Bor combination therapy-mediated cytotoxicity. However, future studies are required to reveal the mechanisms underlying these effects.

In conclusion, the present study demonstrated that AA sensitizes MM cells to Bor-mediated growth inhibition and apoptotic cell death in vitro. Therefore, AA may have potential applications as a chemosensitizer in human cancer treatment. Future in-depth studies, including in vivo experiments, are required to confirm the efficacy of $\mathrm{AA}$ in combination with Bor for MM treatment.

\section{Acknowledgements}

The present study was supported by: The National High Technology Research and Development Program of China (grant no. 2006AA02Z4B5) and the National Natural Science Foundation of China (grant nos. 81272451/H1609 and 81472762/H1609), awarded to J.L.; and the National Natural Science Foundation of China (grant no. 81472390/H1619), General Project from Guangzhou Education Commission (grant no. 1201410188), the Science and Technology Program of Guangzhou (grant no. 201510010127) and the Science and Technology Planning Project of Guangdong Province (grant no. 2014A020212691), awarded to H.H. The authors thank Guangdong Provincial Key Laboratory of Malignant Tumor Epigenetics and Gene Regulation, Sun Yat-Sen Memorial 
Hospital, Sun Yat-Sen University (Guangzhou, China) for assistance with flow cytometry.

\section{References}

1. Sultan S, Irfan SM, Parveen S, Ali H and Basharat M: Multiple Myeloma: A retrospective analysis of 61 patients from a tertiary care center. Asian Pac J Cancer Prev 17: 1833-1835, 2016.

2. Liu JD, Sun CY, Tang L, Wu YY, Wang QY, Hu B and Hu Y: Efficacy and safety of panobinostat in relapsed or/and refractory multiple myeloma: Meta analyses of clinical trials and systematic review. Sci Rep 6: 27361, 2016.

3. Hershko A and Ciechanover A: The ubiquitin system. Annu Rev Biochem 67: 425-479, 1998.

4. Adams J: The development of proteasome inhibitors as anticancer drugs. Cancer Cell 5: 417-421, 2004.

5. Adams J, Palombella VJ, Sausville EA, Johnson J, Destree A Lazarus DD, Maas J, Pien CS, Prakash S and Elliott PJ: Proteasome inhibitors: A novel class of potent and effective antitumor agents. Cancer Res 59: 2615-2622, 1999.

6. Orlowski RZ and Dees EC: The role of the ubiquitination-proteasome pathway in breast cancer: Applying drugs that affect the ubiquitin-proteasome pathway to the therapy of breast cancer. Breast Cancer Res 5: 1-7, 2003.

7. Jagannathan S, Abdel-Malek MA, Malek E, Vad N, Latif T, Anderson KC and Driscoll JJ: Pharmacologic screens reveal metformin that suppresses GRP78-dependent autophagy to enhance the anti-myeloma effect of bortezomib. Leukemia 29: 2184-2191, 2015

8. Eriksson E, Wickström M, Perup LS, Johnsen JI, Eksborg S, Kogner P and Sävendahl L: Protective role of humanin on bortezomib-induced bone growth impairment in anticancer treatment. J Natl Cancer Inst 106: djt459, 2014.

9. Huang WC, Lin YS, Chen CL, Wang CY, Chiu WH and Lin CF Glycogen synthase kinase-3beta mediates endoplasmic reticulum stress-induced lysosomal apoptosis in leukemia. J Pharmacol Exp Ther 329: 524-531, 2009.

10. Jiang C, Zhang S, Liu H, Zeng Q, Xia T, Chen Y, Kuang G, Zhao G, Wu X, Zhang X, et al: The role of the IRE1 pathway in PBDE-47-induced toxicity in human neuroblastoma SH-SY5Y cells in vitro. Toxicol Lett 211: 325-333, 2012.

11. Ron D and Walter P: Signal integration in the endoplasmic reticulum unfolded protein response. Nat Rev Mol Cell Biol 8: 519-529, 2007.

12. Sano R and Reed JC: ER stress-induced cell death mechanisms Biochim Biophys Acta 1833: 3460-3470, 2013

13. Xu C, Bailly-Maitre B and Reed JC: Endoplasmic reticulum stress: Cell life and death decisions. J Clin Invest 115 2656-2664, 2005.

14. Armstrong JL, Flockhart R, Veal GJ, Lovat PE and Redfern CP: Regulation of endoplasmic reticulum stress-induced cell death by ATF4 in neuroectodermal tumor cells. J Biol Chem 285 : 6091-6100, 2010

15. Jiang HY and Wek RC: Phosphorylation of the alpha-subunit of the eukaryotic initiation factor-2 (eIF2alpha) reduces protein synthesis and enhances apoptosis in response to proteasome inhibition. J Biol Chem 280: 14189-14202, 2005.

16. Qing G, Li B, Vu A, Skuli N, Walton ZE, Liu X, Mayes PA, Wise DR, Thompson CB, Maris JM, et al: ATF4 regulates MYC-mediated neuroblastoma cell death upon glutamine deprivation. Cancer Cell 22: 631-644, 2012

17. Jung J, Kim J, Roh SH, Jun I, Sampson RD, Gee HY, Choi JY and Lee MG: The HSP70 co-chaperone DNAJC14 targets misfolded pendrin for unconventional protein secretion. Nat Commun 7 : 11386, 2016.

18. Wu Y, He L, Zhang L, Chen J, Yi Z, Zhang J, Liu M and Pang X: Anacardic acid (6-pentadecylsalicylic acid) inhibits tumor angiogenesis by targeting Src/FAK/Rho GTPases signaling pathway. J Pharmacol Exp Ther 339: 403-411, 2011.

19. Seong YA, Shin PG and Kim GD: Anacardic acid induces mitochondrial-mediated apoptosis in the A549 human lung adenocarcinoma cells. Int J Oncol 42: 1045-1051, 2013.
20. Seong YA, Shin PG, Yoon JS, Yadunandam AK and Kim GD: Induction of the endoplasmic reticulum stress and autophagy in human lung carcinoma A549 cells by anacardic acid. Cell Biochem Biophys 68: 369-377, 2014.

21. Huang H, Hua X, Liu N, Li X, Liu S, Chen X, Zhao C, Lan X, Yang C, Dou QP, et al: Anacardic acid induces cell apoptosis associated with induction of ATF4-dependent endoplasmic reticulum stress. Toxicol Lett 228: 170-178, 2014

22. Hill DS, Martin S, Armstrong JL, Flockhart R, Tonison JJ, Simpson DG, Birch-Machin MA, Redfern CP and Lovat PE: Combining the endoplasmic reticulum stress-inducing agents bortezomib and fenretinide as a novel therapeutic strategy for metastatic melanoma. Clin Cancer Res 15: 1192-1198, 2009.

23. Huang H, Liu N, Guo H, Liao S, Li X, Yang C, Liu S, Song W, Liu C, Guan L, et al: L-carnitine is an endogenous HDAC inhibitor selectively inhibiting cancer cell growth in vivo and in vitro. PLoS One 7: e49062, 2012.

24. Huang H, Zhang X, Li S, Liu N, Lian W, McDowell E, Zhou P, Zhao C, Guo H, Zhang C, et al: Physiological levels of ATP negatively regulate proteasome function. Cell Res 20: 1372-1385, 2010.

25. Liu N, Li X, Huang H, Zhao C, Liao S, Yang C, Liu S, Song W, Lu X, Lan X, et al: Clinically used antirheumatic agent auranofin is a proteasomal deubiquitinase inhibitor and inhibits tumor growth. Oncotarget 5: 5453-5471, 2014.

26. Huang H, Chen D, Li S, Li X, Liu N, Lu X, Liu S, Zhao K, Zhao $\mathrm{C}$, Guo $\mathrm{H}$, et al: Gambogic acid enhances proteasome inhibitor-induced anticancer activity. Cancer Lett 301: 221-228, 2011.

27. Chou TC and Talalay P: Quantitative analysis of dose-effect relationships: The combined effects of multiple drugs or enzyme inhibitors. Adv Enzyme Regul 22: 27-55, 1984.

28. Wang Q, Li J, Gu J, Huang B, Zhao Y, Zheng D, Ding Y and Zeng L: Potentiation of (-)-epigallocatechin-3-gallate-induced apoptosis by bortezomib in multiple myeloma cells. Acta Biochim Biophys Sin (Shanghai) 41: 1018-1026, 2009.

29. Ma C, Mandrekar SJ, Alberts SR, Croghan GA, Jatoi A, Reid JM, Hanson LJ, Bruzek L, Tan AD, Pitot HC, et al: A phase I and pharmacologic study of sequences of the proteasome inhibitor, bortezomib (PS-341, Velcade), in combination with paclitaxel and carboplatin in patients with advanced malignancies. Cancer Chemother Pharmacol 59: 207-215, 2007.

30. Fribley A, Zeng Q and Wang CY: Proteasome inhibitor PS-341 induces apoptosis through induction of endoplasmic reticulum stress-reactive oxygen species in head and neck squamous cell carcinoma cells. Mol Cell Biol 24: 9695-9704, 2004.

31. Nawrocki ST, Carew JS, Dunner K Jr, Boise LH, Chiao PJ, Huang P, Abbruzzese JL and McConkey DJ: Bortezomib inhibits PKR-like endoplasmic reticulum (ER) kinase and induces apoptosis via ER stress in human pancreatic cancer cells. Cancer Res 65: 11510-11519, 2005.

32. Zhao X, Qiu W, Kung J, Zhao X, Peng X, Yegappan M, Yen-Lieberman B and Hsi ED: Bortezomib induces caspase-dependent apoptosis in Hodgkin lymphoma cell lines and is associated with reduced c-FLIP expression: A gene expression profiling study with implications for potential combination therapies. Leuk Res 32: 275-285, 2008.

33. Shringarpure R, Catley L, Bhole D, Burger R, Podar K, Tai YT, Kessler B, Galardy P, Ploegh H, Tassone P, et al: Gene expression analysis of B-lymphoma cells resistant and sensitive to bortezomib. Br J Haematol 134: 145-156, 2006.

34. Calvaruso G, Giuliano M, Portanova P, Pellerito O, Vento R and Tesoriere G: Hsp72 controls bortezomib-induced HepG2 cell death via interaction with pro-apoptotic factors. Oncol Rep 18: 447-450, 2007.

35. Qi W, White MC, Choi W, Guo C, Dinney C, McConkey DJ and Siefker-Radtke A: Inhibition of inducible heat shock protein-70 (hsp72) enhances bortezomib-induced cell death in human bladder cancer cells. PLoS One 8: e69509, 2013.

36. Beck D, Niessner H, Smalley KS, Flaherty K, Paraiso KH, Busch C, Sinnberg T, Vasseur S, Iovanna JL, Driessen S, et al: Vemurafenib potently induces endoplasmic reticulum stress-mediated apoptosis in BRAFV600E melanoma cells. Sci Signal 6: ra7, 2013. 